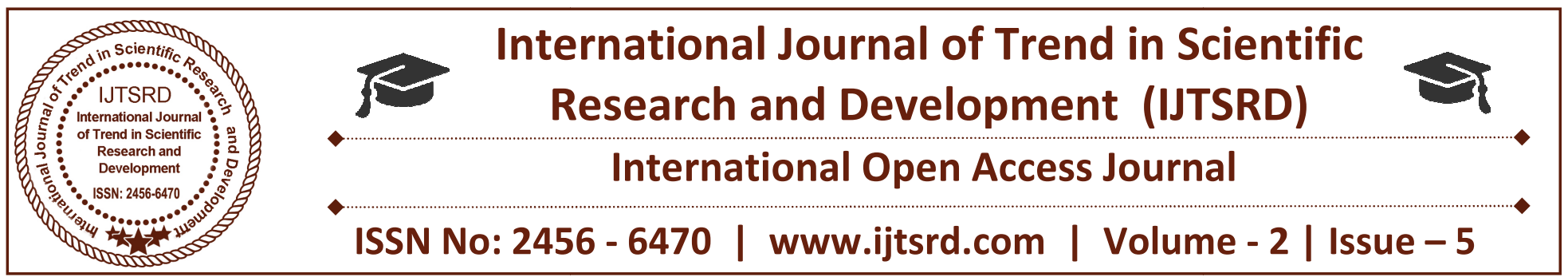

\title{
UV-Visible Spectrophotometric Method of Paracetamol Tablet Formulation
}

\author{
Selvi S R \\ Lecturer Senior Grade, Department of Physics, Muthiah Polytechnic College, \\ Annamalai Nagar, Chidambaram, Tamil Nadu, India
}

\begin{abstract}
A novel, safe and sensitive method of spectrophotometric estimation in UV-region has been developed for the assay of Paracetamol in its tablet formulation. The method have been developed and validated for the assay of Paracetamol using Methanol and water as diluents. Which does not shows any interference in spectrophotometric estimations. All the parameters of the analysis were chosen according to ICH [Q2 (R1)] guideline and validated statistically using RSD and \%RSD along with neat chromate grams.
\end{abstract}

Keyword: UV Spectrography, Paracetamol, Concentration, Tablets

\section{INTRODUCTION}

Spectroscopic techniques are increasingly proposed as alternative methods for the quantification of APIs in pharmaceuticals. This is due to their advantages over the traditional techniques, such as fast, in-line, noninvasive and non-destructive measurements without the need of sample preparation. Near infrared (NIR) and Raman spectroscopy have been identified as effective PAT-tools for real-time measurements of critical process and product attributes during pharmaceutical processing. Raman spectroscopy is until now mostly applied for solid dosage forms [1e6]. Some in-line quantitative applications for hot-melt extrusion processes have also been reported [7e9]. Raman spectroscopy has an added value for quantification purposes of pharmaceutical formulations where water is present, such as in semisolid and liquid formulations, since water produces almost no Raman signal. Research has already been conducted to investigate the opportunity offered by Raman spectroscopy for these formulations [10e15], however less frequently as an in-line analytical tool [16]. For some applications, these spectroscopic techniques are not feasible, such as those that require the quantification of low-dosed analytics. Fluorescence spectroscopy can be an alternative to the conventional spectroscopic techniques for these applications because of its high sensitivity and detection sensitivity. A drawback of fluorescence spectroscopy is that the analytic needs to be a native fluorophore in order to detect it, which limits the number of possible applications for this technique. UV spectroscopy is a widely used quantitative analytical technique that finds its application in many research domains and is capable of quantifying very low concentrations $(<0.01 \%)$ [20e24]. Nevertheless, studies describing on-line and in-line applications of UV/VIS spectroscopy with fibre-optic probes are limited. O'Keeffeet al. monitored the ozone concentration of a gas in an aluminium glass cell with a fibre-based UV/VIS spectroscopy system . Quinn et al. followed the reaction of a nucleoside with trityl chloride in pyridine in a liquid environment, using a fibre-optic transmission probe. The concentration of starting material and product was predicted via a PLS regression model. Furthermore, a mixing study using a fibre-optic UV/VIS monitoring technique was reported by $\mathrm{Ng}$ and Assirelli. In this paper, bromophenol blue sodium salt was used as a nonreactive tracer in distilled water. A good agreement between the UV/VIS technique and the traditional conductivity technique was found. Other examples of on-line and in-line UV spectroscopic applications in literature are drug dissolution tests, where the drug release was monitored in real-time. However, the use of UV spectroscopy for in-line monitoring of critical quality attributes during pharmaceutical 
manufacturing processes of semi-solids and liquids is not yet described in literature. This work deals with the validation of the developed method for the assay of Paracetamol from its dosage form (tablets). Hence, the method can be used for routine quality control analysis and also stability.

Paracetamol or acetaminophen is a widely used overthe-counter analgesic (pain reliever) and antipyretic (fever reducer). It is commonly used for the relief of headaches and other minor aches and pains and is a major ingredient in numerous cold and flu remedies. In combination with opioid analgesics, Paracetamol can also be used in the management of more severe pain such as post-surgical pain and providing palliative care in advanced cancer patients [5]. The onset of analgesia is approximately 11 minutes after oral administration of Paracetamol [6], and its halflife is 1-4 hours. Though acetaminophen is used to treat inflammatory pain, it is not generally classified as an NSAID because it exhibits only weak antiinflammatory activity. While generally safe for use at recommended doses (1,000 $\mathrm{mg}$ per single dose and up to $4,000 \mathrm{mg}$ per day for adults), acute overdoses of Paracetamol can cause potentially fatal liver damage and, in rare individuals, a normal dose can do the same; the risk is heightened by alcohol consumption. Paracetamol toxicity is the foremost cause of acute liver failure in the Western world, and accounts for most drug overdoses in the United States, the United Kingdom, Australia and New Zealand [7-10]. It is the active metabolite of Phenacetin, once popular as an analgesic and antipyretic in its own right, but unlike Phenacetin and its combinations, Paracetamol is not considered carcinogenic at therapeutic doses [11]. The words acetaminophen (used in the United States, Canada, Japan, South Korea, Hong Kong, and Iran [12] and Paracetamol (used elsewhere) both come from a chemical name for the compound: paraacetylaminophenol and para-acetylaminophenol. In some contexts, it is simply abbreviated as APAP, for acetyl-paraaminophenol.

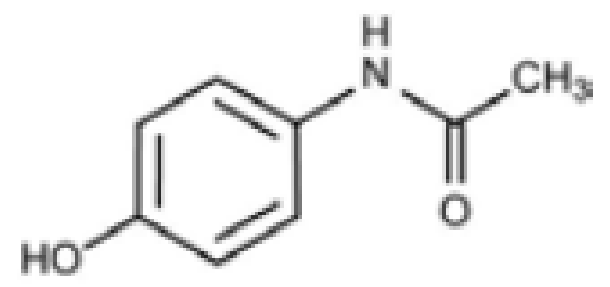

Paracetamol is part of the class of drugs known as "aniline analgesics"; it is the only such drug still in use today. It is not considered an NSAID because it does not exhibit significant antiinflammatory activity (it is a weak COX inhibitor). This is despite the evidence that Paracetamol and NSAIDs have some similar pharmacological activity.

To date, the mechanism of action of Paracetamol is not completely understood. The main mechanism proposed is the inhibition of cyclooxygenase (COX), and recent findings suggest that it is highly selective for COX-2. While it has analgesic and antipyretic properties comparable to those of aspirin or other NSAIDs, its peripheral anti-inflammatory activity is usually limited by several factors, one of which is the high level of peroxides present in inflammatory lesions. However, in some circumstances, even peripheral anti-inflammatory activity comparable to other NSAIDs can be observed. An article [18] in Nature Communications from researchers in London, UK and Lund, Sweden in November 2011 has found a hint to the analgesic mechanism of acetaminophen (Paracetamol), being that the metabolites of acetaminophen e.g. NAPQI, act on TRPA1-receptors in the spinal cord to suppress the signal transduction from the superficial layers of the dorsal horn, to alleviate pain.

Paracetamol is metabolised primarily in the liver, into non-toxic products. Three metabolic pathways are no table:

$>$ Glucuronidation is believed to account for $40 \%$ to two-thirds of the metabolism of paracetamol.

Sulfation (sulfate conjugation) may account for $20-40 \%$.

$\mathrm{N}$-hydroxylation and rearrangement, then GSH conjugation, accounts for less than $15 \%$. The hepatic cytochrome P450 enzyme system metabolizes paracetamol, forming a minor yet significant alkylating metabolite known as NAPQI (N-acetyl-p-benzo-quinone imine). NAPQI is then irreversibly conjugated with the sulfhydryl groups of glutathione [20]. All three pathways yield final products that are inactive, nontoxic, and eventually excreted by the kidneys. In the third pathway, however, the intermediate product NAPQI is toxic. NAPQI is primarily responsible for the toxic effects of Paracetamol; this constitutes an example of toxication.

The discussion of the validation of analytical procedures is directed to the four most common types of analytical procedures:

$>$ Identification tests; 
$>$ Quantitative tests for impurities' content;

$>$ Limit tests for the control of impurities; Quantitative tests of the active moiety in samples of drug substance or drug product or other selected component(s) in the drug product.

The aim and scope of the proposed work are as under:

$>$ To develop suitable spectrophotometric method for assay of Paracetamol tablet.

$>$ Perform the validation for the method.

\section{Experimental methods}

Paracetamol standard of was provided by Torque Pharmaceuticals (P) Ltd. (India). Paracetamol tablets containing $500 \mathrm{mg}$ Paracetamol and the inactive ingredient used in drug matrix were obtained from market. Analytical grade methanol and water were obtained from Spectrochem Pvt. Ltd., Mumbai (India).

Diluent preparation is the using Methanol and water $(15: 85, \mathrm{v} / \mathrm{v})$ used as a diluent. Standard preparation 10 $\mathrm{mg}$ drug was dissolved in $15 \mathrm{ml}$ methanol and was shaken well. Then $85 \mathrm{ml}$ water was added to it to adjust the volume up to $100 \mathrm{ml}(100 \mathrm{ppm})$. From that $5 \mathrm{ml}$ was taken and volume was adjusted up to $50 \mathrm{ml}$ with diluents.

20 tablets were weighed and powdered. Powdered tablet equivalentto $100 \mathrm{mg}$ of paracetamol was weighed and taken into $100 \mathrm{ml}$ volumetric flask then $15 \mathrm{ml}$ of methanol was added and shaken well to dissolve it after that $85 \mathrm{ml}$ of water was added to adjust the volume up to $100 \mathrm{ml}$. From that $1 \mathrm{ml}$ of solution was withdrawn and taken in $100 \mathrm{ml}$ volumetric flask. The volume was adjusted with diluent up to $100 \mathrm{ml}$. UV-Visible double beam spectrophotometer with matched quartzcells $(1 \mathrm{~cm})$ Evolution 201 model is used

UV-Visible spectrophotometry is one of the most frequently employed technique in pharmaceutical analysis. It involves measuring the amount of ultraviolet or visible radiation absorbed by a substance in solution. Instrument which measure the ratio, or function of ratio, of the intensity of two beams of light in the U.V-Visible region are called Ultraviolet-Visible spectrophotometers. In qualitative analysis, organic compounds can be identified by use of spectrophotometer, if any recorded data is available, and quantitative spectrophotometric analysis is used to ascertain the quantity of molecular species absorbing the radiation. Spectrophotometric technique is simple, rapid, moderately specific and applicable to small quantities of compounds. The fundamental law that governs the quantitative spectrophotometric analysis is the Beer -Lambert law. Beer's law: It states that the intensity of a beam of parallel monochromatic radiation decreases exponentially with the number of absorbing molecules. In other words, absorbance is proportional to the concentration. Lambert's law: It states that the intensity of a beam of parallel monochromatic radiation decreases exponentially as it passes through a medium of homogeneous thickness. A combination of these two laws yields the Beer-Lambert law.

Beer-Lambert law: When beam of light is passed through a transparent cell containing a solution of anabsorbing substance, reduction of the intensity of light may occur.

Validation is concerned with assuring that a measurement process produces valid measurements. • Results from method validation can be used to judge the quality, reliability and consistency of analytical results. It is an integral part of any good analytical practice. - A measurement process producing valid measurements for an intended application is fit for purpose

Method validation is the process used to confirm that the analytical procedure employed for a specific test is suitable for its intended use. Results from method validation can be used to judge the quality, reliability and consistency of analytical results; it is an integral part of any good analytical practice.

\section{Results and Discussion}

Proper wave length selection of the methods depends upon the nature of the sample and its solubility. To develop a rugged and suitable spectrophotometric method for the quantitative determination of paracetamol, the analytical condition were selected after testing the different parameters such as diluents, buffer, buffer concentration, and other chromatographic conditions. Our preliminary trials were by using different compositions of diluents consisting of water with buffer and methanol. By using diluent consisted of methanol - water (50:50, $\mathrm{v} / \mathrm{v}$ ) best result was obtained and degassed in an ultrasonic bath (Enertech Electronics Private 
Limited). Below figures represent the spectrums of blank, standard and test preparation respectively.
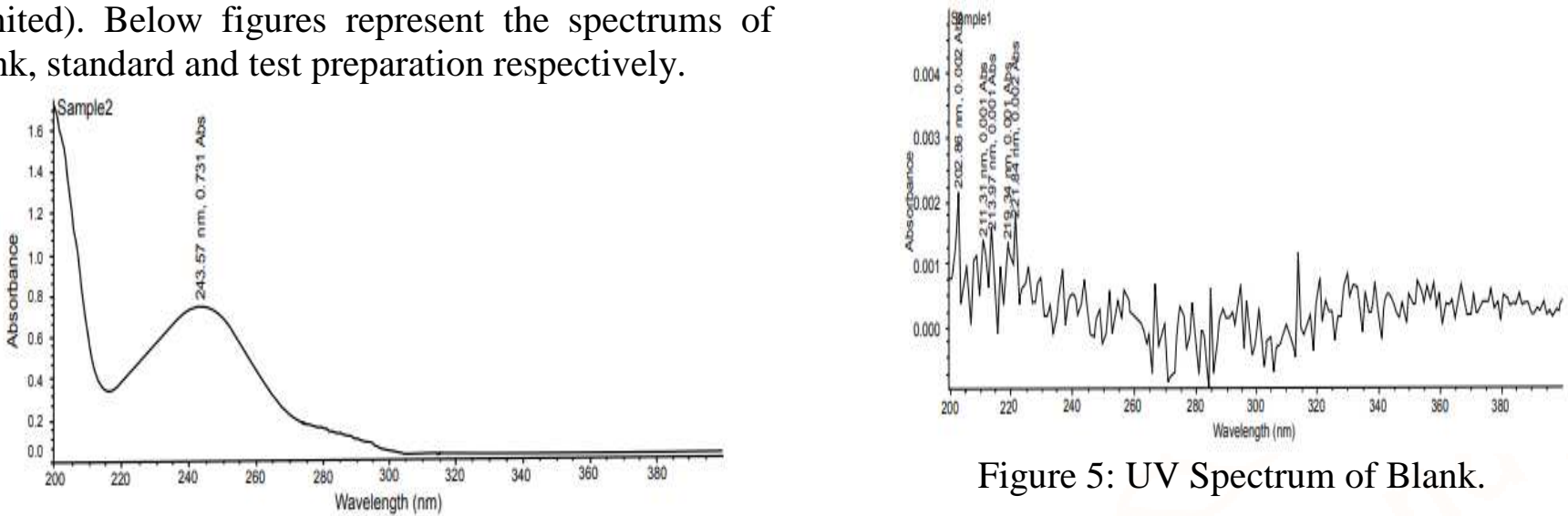

Figure 5: UV Spectrum of Blank.

Figure 1: UV spectrum of Paracetamol in Standard solution.

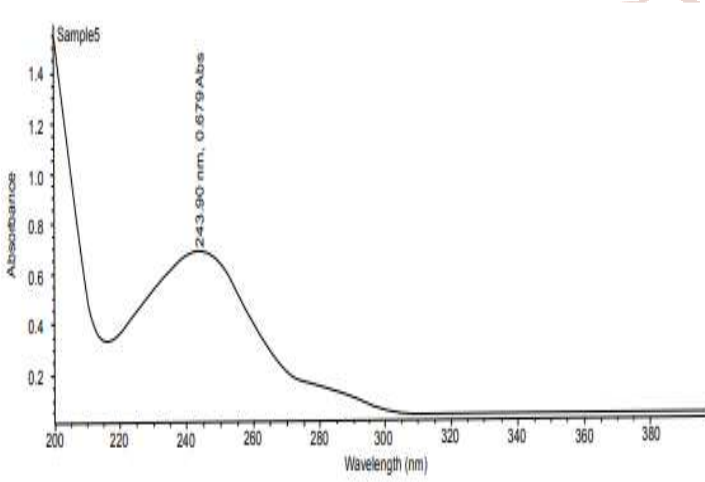

Figure 2: UV spectrum of Paracetamol in Sample solution.

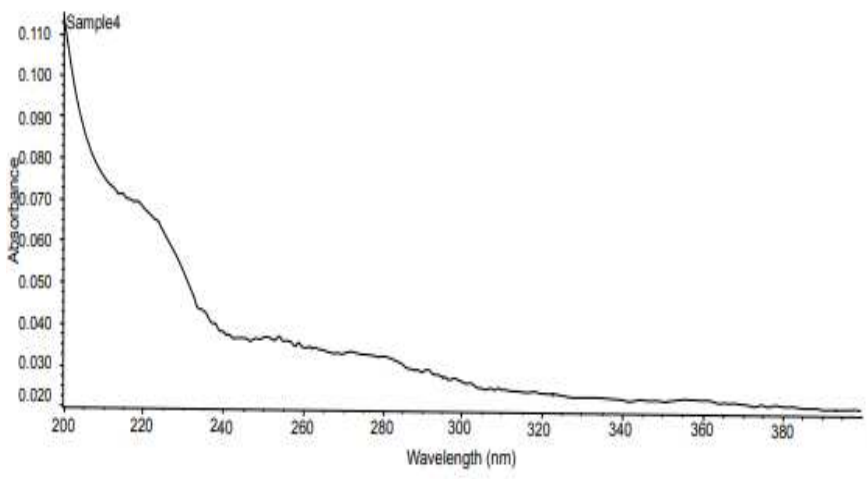

Figure 3: UV Spectrum of Placebo.

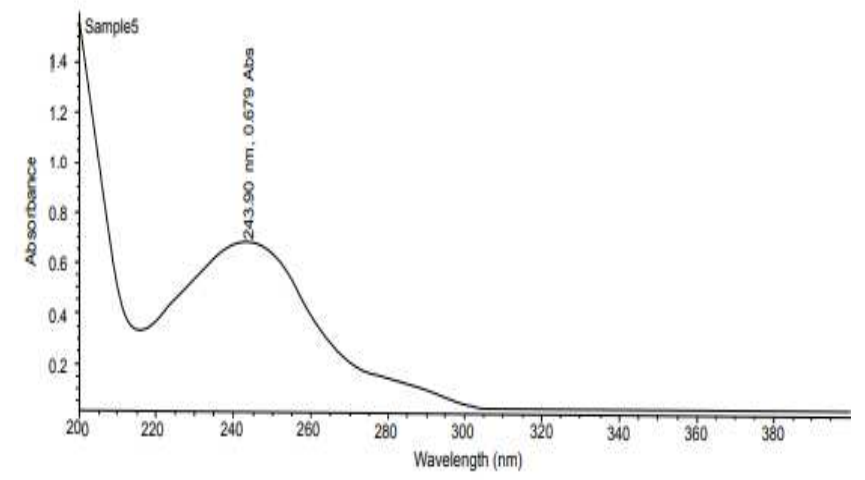

Figure 4: UV Spectrum of Placebo+analyte.

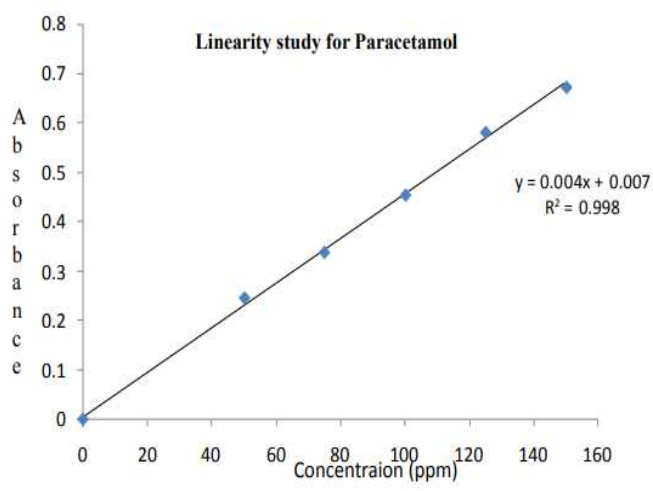

Figure 6: Linearity curve for Paracetamol.

Specificity: Resolution of the analyte peak from the nearest peak: Solution of each of the analyte was injected separately and their retention time is noted. The standard working solution containing a mixture of the component being analyze is also injected and each of analyte peaks is check for its resolution from the nearest (Figures 2,3,4,5).

Linearity: Six points calibration curve were obtained in a concentration range from $0-150 \mathrm{ppm}$ for Paracetamol. The response of the drug was found to be linear in the investigation concentration range and the linear regression equation was $\mathrm{y}=0.004 \mathrm{x}+0.007$ with correlation coefficient 0.998 (Figure 6).

Precision: Precision of the analytical method is ascertained by carrying out the analysis as per the procedure and as per normal weight taken for analysis. Repeat the analysis six times. Calculate the $\%$ assay, mean assay, \% Deviation and \% relative standard deviation and \%RSD. The developed method was found to be precise as the \% RSD values for the repeatability and intermediate precision studies were $<0.98 \%$ and $<0.79 \%$, respectively.

\section{Accuracy}

Accuracy of the method is ascertained by standard addition method at 3 levels. Standard quantity 
equivalent to $50 \%, 100 \%$ and $125 \%$ is to be added in sample. The result shown that best recoveries (98.54$99.13 \%$ ) of the spiked drug were obtained at each added concentration, indicating that the method was accurate. Composition of Placebo Added for Accuracy Study.

\section{Solution stability study}

Results obtain in the solution stability study at different time intervals for test preparation. It was concluded that the test preparation solution was found stable up to $8 \mathrm{hr}$ at room temperature, as during this time the result was not decrease below the minimum percentage.

\section{Robustness}

The evaluation of robustness should be considered during the development phase and depends on the type of procedure conditions, assay value of the test preparation solution was not affected and it was in accordance with that of actual. System suitability parameters were also found satisfactory; hence the analytical method would be concluded as robust.

\section{System suitability}

A system suitability test of the spectrophotometric system was performed before each validation run. Six replicate reading of standard preparation were taken and \%RSD of standard reading were taken for same. Acceptance criteria for system suitability, \%RSD of standard reading not more than $2.0 \%$, were full fill during all validation parameter.

\section{Conclusion}

The present analytical method was validated as per ICH Q2(R1) guideline and it meets to specific acceptance criteria. It is concluded that the analytical method was specific, precise, linear, accurate, robust and having stability indicating characteristics. The present analytical method can be used for its intended purpose.

\section{References}

1. Willard-Hobart H, Merritt Jr Lynne L, Dean John A (1974) Instrumental Methods of Analysis. (5thedn), Von Nostrand, University of Michigan.
2. ChatwalGR, Anand S (2002) Instrumental Methods of Chemical Analysis. (5thedn), Himalaya Publishing House, New Delhi.

3. Davidson AG (2002) Ultraviolet-visible absorption spectrophotometry. In BeckettAH, Stenlake JB, (4thedn), Practical Pharmaceutical chemistry. CBS Publishers and distributors, New Delhi, 275-278.

4. Patil KM, Bodhankar SL (2005) Highperformance thin-layer chromatographic determination of lamotrigine in serum. $\mathrm{J}$ Chromatogr B AnalytTechnol Biomed Life Sci 823: $152-157$.

5. Scottish Intercollegiate Guidelines Network (SIGN) (2008) Control of pain in adults with cancer-A national clinical guideline. Scotland: National Health Service (NHS).

6. Moller P, Sindet-Pedersen S, Petersen CT, Juhl GI, Dillenschneider A, et al. (2005) Onset of acetaminophen analgesia: comparison of oral and intravenous routes after third molar surgery. $\mathrm{Br} \mathbf{J}$ Anaesth 94: 642-648.

7. Daly FF, Fountain JS, Murray L, Graudins A, Buckley NA (2008) Guidelines for the management of paracetamol poisoning in Australia and New Zeal and explanation and elaboration. A consensus statement from clinical toxicologists consulting to the Australasian poisons information centres. Med J Aust 188: 296301.

8. Khashab M, Tector AJ, Kwo PY (2007) Epidemiology of acute liver failure. CurrGastroenterol Rep 9: 66-73.

9. Hawkins LC, Edwards JN, Dargan PI (2007) Impact of restricting paracetamol pack sizes on paracetamol poisoning in the United Kingdom: a review of the literature. Drug Saf 30: 465-479.

10. Larson AM, Polson J, Fontana RJ, Davern TJ, Lalani E, et al. (2005)Acetaminophen-induced acute liver failure: results of a United States multicenter, prospective study. Hepatology 42: 1364-1372. 